\title{
OTIMIZAÇÃO DOS PARÂMETROS DE EXTRAÇÃO PARA DETERMINAÇÃo MULTIRESÍDUO DE PESTICIDAS EM AMOSTRAS DE ÁGUA EMPREGANDO MICROEXTRAÇÃO EM FASE SÓLIDA
}

\author{
Emy Komatsu e Jorge Moreira Vaz* \\ Centro de Química e Meio Ambiente, Instituto de Pesquisas Energéticas e Nucleares, Av. Prof. Lineu Prestes, 2242, \\ 05508-000 São Paulo - SP
}

Recebido em 18/9/03; aceito em 18/3/04; publicado na web em 19/7/04

\begin{abstract}
PARAMETER OPTIMIZATION FOR PESTICIDE MULTIRESIDUE DETERMINATION USING SPME IN WATER SAMPLES. In this study the factors that affect the extraction of pesticide residues from water samples for their determination by solid-phase micro-extraction (SPME) and GC/MS were optimized. The fiber polymer, the extraction mode, the ionic strength, the stirring rate, the $\mathrm{pH}$ and the extraction time were the most significant parameters evaluated. The recovery obtained with the proposed method ranged from 94 to $110 \%$ and the sensitivity of the method, determined by calculating the detection limit (DL), ranged from 0.004 to $0.1 \mu \mathrm{g} \mathrm{l}^{-1}$. The results obtained with spiked real samples, at the $0.1 \mu \mathrm{g} \mathrm{l^{-1 }}$ level, showed acceptable conformity with the expected values.
\end{abstract}

Keywords: SPME; pesticides; GC-MS.

\section{INTRODUÇÃO}

Pesticidas são compostos orgânicos de origem antrópica intensamente empregados em atividades agrícolas, possuem alta toxicidade e geralmente são apontados como causadores de muitos danos à saúde humana, à flora e à fauna ${ }^{1-3}$

Segundo a $\mathrm{EPA}^{4}$, um pesticida é qualquer substância ou mistura de substâncias com capacidade de prevenir, destruir, repelir ou atenuar qualquer peste. Os pesticidas podem ser classificados de acordo com o tipo de peste - algicidas, fungicidas, herbicidas, inseticidas, acaricidas, moluscidas, nematicidas, ovicidas, entre outros. Além disso podem ser incluídas substâncias desfolhantes, dessecantes ou reguladoras do crescimento de insetos e plantas ${ }^{4}$.

Os pesticidas também podem ser classificados de acordo com sua estrutura química. A escolha dos pesticidas estudados neste trabalho foi fundamentada, principalmente, em sua grande utilização em escala mundial e nacional, além de seu elevado potencial xenobiótico, tendo alguns estudos já apontado para a ubiqüidade de alguns deles ${ }^{5}$. Suas características gerais ${ }^{6}$, incluindo a classificação de acordo com a toxicidade ${ }^{4}$ são apresentadas na Tabela 1.

Devido a sua persistência os pesticidas podem ser encontrados nos diferentes compartimentos ambientais ${ }^{7,8}$. No ar, são originários dos procedimentos de pulverização na forma de aerossóis. No solo, são encontrados devido a derramamentos ou descartes inadequados, que por percolação podem atingir lençóis de águas subterrâneas. Em águas superficiais são originários pelo carregamento através de chuvas, derramamentos ou usos em campanhas de saúde pública.

No caso de amostras aquosas, a determinação de pesticidas usualmente é precedida por etapas de extração e pré-concentração antes de se efetuar a quantificação. Dentre as técnicas de extração ${ }^{1,5,7,9}$ mais empregadas podem ser destacadas a extração líquido-líquido (LLE) e a extração em fase sólida (SPE). Para a quantificação normalmente são empregadas técnicas de separação ${ }^{8-10}$ e dentre as mais comuns para amostras ambientais podem ser destacadas a cromatografia à gás (GC) e a cromatografia líquida de alto desempenho (HPLC).

Além dos procedimentos de extração citados, outra técnica em ascensão é a microextração em fase sólida ${ }^{8,11-15}$ (SPME). Esta consiste em duas etapas básicas; a adsorção e a desorção dos compostos em uma fibra de sílica fundida recoberta com material polimérico. A etapa de adsorção é realizada expondo a fibra diretamente na amostra ou em sua fase gasosa e a desorção ocorre no próprio injetor do cromatógrafo a gás (ou no injetor de um HPLC).

A SPME difere das outras técnicas de extração para amostras aquosas pelo menor tempo de análise e pela não utilização de solventes, minimizando a geração de descartes e a exposição ocupacional. Em relação às outras, é uma técnica simples, rápida e de fácil

Tabela 1. Características gerais dos compostos estudados

\begin{tabular}{lcccccc}
\hline Pesticida & CAS & Fórmula & Grupo & Uso $^{\text {a) }}$ & Classe $^{\text {b) }}$ & Solubilidade $^{\text {c) }}\left(m^{-1}\right)$ \\
\hline Trifluralina & $1582-09-8$ & $\mathrm{C}_{13} \mathrm{H}_{16} \mathrm{~F}_{3} \mathrm{~N}_{3} \mathrm{O}_{4}$ & Dinitroanilina & $\mathrm{H}$ & III & 0,22 \\
Simazina & $122-34-9$ & $\mathrm{C}_{7} \mathrm{H}_{12} \mathrm{ClN}_{5}$ & Triazina & $\mathrm{H}$ & III & 6,2 \\
Atrazina & $1912-24-9$ & $\mathrm{C}_{8} \mathrm{H}_{14} \mathrm{ClN}_{5}$ & Triazina & $\mathrm{H}$ & $\mathrm{III}$ & 33 \\
Pentaclorofenol & $131-52-2$ & $\mathrm{C}_{6} \mathrm{Cl}_{5} \mathrm{NaO}_{2}$ & Organoclorado & $\mathrm{H} ; \mathrm{I} ; \mathrm{F}$ & $\mathrm{I}$ & 80 \\
Bifentrina & $82657-04-3$ & $\mathrm{C}_{23} \mathrm{H}_{22} \mathrm{CF}_{3} \mathrm{O}_{2}$ & Piretróide & $\mathrm{I} ; \mathrm{A}$ & II & 0,1 \\
Permetrina & $52645-53-1$ & $\mathrm{C}_{21} \mathrm{H}_{2} \mathrm{OCl}_{2} \mathrm{O}_{3}$ & Piretróide & I & III & 0,2 \\
\hline
\end{tabular}

a) $\mathrm{A}=$ Acaricida; $\mathrm{F}=$ Fungicida; $\mathrm{H}=$ Herbicida; $\mathrm{I}$ = Inseticida; b) Classe I - Produtos Extremamente Tóxicos (faixa vermelha); Classe II Produtos Altamente Tóxicos (faixa amarela); Classe III - Produtos Medianamente Tóxicos (faixa azul); Classe IV - Produtos Pouco Tóxicos (faixa verde); c) em água a $20^{\circ} \mathrm{C}$ 
manuseio, além da vantagem da utilização de pequena quantidade de amostra, podendo ser aplicada a diversas áreas, tais como farmacêutica, ambiental, alimentícia, aromas, forense e toxicológica ${ }^{11-13,16-}$ ${ }^{21}$. Entretanto, embora seja uma técnica versátil, as condições de extração devem ser otimizadas para cada tipo de amostra ${ }^{22}$. Neste caso, os principais parâmetros a serem avaliados são modo de extração ("headspace" ou imersão direta na amostra líquida), tipo de fibra, $\mathrm{pH}$, velocidade de agitação, concentração iônica, temperatura e volume da amostra.

O objetivo deste trabalho consiste em desenvolver metodologia analítica multiresíduo para determinação dos pesticidas trifluralina, simazina, atrazina, pentaclorofenol, bifentrina, cis-permetrina e transpermetrina, em amostras de água, empregando microextração em fase sólida (SPME), seguida pela determinação por GC/MS.

\section{PARTE EXPERIMENTAL}

\section{Reagentes}

Os padrões dos pesticidas trifluralina, simazina, atrazina e bifentrina foram obtidos do "Institute of Organic Industrial Chemistry"; pentaclorofenol, da Supelco; cis-permetrina do "Laboratory of the Government Chemist" e trans-permetrina da "Riedel-de-Häen". Soluções estoque individuais $1000 \mu \mathrm{g} \mathrm{mL} \mathrm{m}^{-1}$ foram preparadas dissolvendo-se a quantidade apropriada dos padrões em acetonitrila (grau HPLC, J. T. Baker) e armazenadas a $4{ }^{\circ} \mathrm{C}$. Essas soluções foram utilizadas para preparar soluções padrão intermediárias, trabalho e para o "spike" em água. A água deionizada foi obtida por um sistema Milli-Q (Barnstead). O sulfato de sódio (anidro, P.A.), hidróxido de sódio (pastilhas, P.A.) e ácido clorídrico (37\%) foram obtidos da Merck. A amostra de água bruta foi coletada no município de Cananéia, no Rio Itapitangui, com ponto de coleta antes da estação de tratamento de água, classificada como corpo d'água ${ }^{23}$ de Classe 2.

\section{Procedimentos de extração com SPME}

Foram avaliadas quatro fibras com diferentes recobrimentos poliméricos PDMS, (polidimetilsiloxano), CAR/PDMS (carboxen/ polidimetilsiloxano), CW/DVB, (carbowax/divinilbenzeno) e PA (poliacrilato), todas marca Supelco. Antes do uso, as fibras foram condicionadas no injetor do cromatógrafo de acordo com tempo e temperatura recomendados no manual do fabricante, específicos para cada tipo de fibra.

Para a extração, inicialmente a fibra foi colocada em contato com a amostra (modo direto ou "headspace"), onde ocorre a adsorção dos analitos no recobrimento polimérico da fibra. A seguir, a fibra foi inserida diretamente no injetor de um cromatógrafo a gás, a $260^{\circ} \mathrm{C}$, onde ocorreu a desorção térmica dos analitos retidos no recobrimento polimérico.

\section{Análise por GC/MS}

As determinações foram efetuadas utilizando-se um GC-MS modelo QP5000 marca Shimadzu equipado com injetor tipo "split/ splitless" a $260^{\circ} \mathrm{C}$. Os analitos foram separados em uma coluna capilar DB-5MS (30 m x 0,25 mm) com fase estacionária de 0,25 $\mu \mathrm{m}$ de espessura (J\&W Scientific). A temperatura da interface foi de $240{ }^{\circ} \mathrm{C}$ e foi utilizado hélio $(99,999 \%)$ como gás de arraste, com

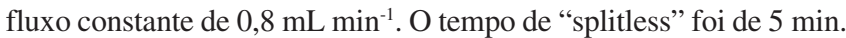
O programa de temperatura para aquecimento do forno foi temperatura inicial de $40^{\circ} \mathrm{C}$, durante $5 \mathrm{~min}$, programado para $70^{\circ} \mathrm{C}$ em uma razão de $40{ }^{\circ} \mathrm{C} / \mathrm{min}$ e de 70 a $325^{\circ} \mathrm{C}$ na razão de $10^{\circ} \mathrm{C} / \mathrm{min}$, manten- do a temperatura de $325^{\circ} \mathrm{C}$ por 5 min. A aquisição do sinal foi efetuada tanto nos modos "full scan" e SIM ("selected ion monitoring") empregando-se impacto eletrônico. A tensão da eletromultiplicadora empregada no modo "full scan" foi de $1,60 \mathrm{kV}$ e no modo SIM de $2,50 \mathrm{kV}$. As curvas analíticas para todos os analitos foram obtidas empregando-se a SPME com seis níveis de concentrações, em triplicata, na faixa de 0,12 a $10,88 \mu \mathrm{g} \mathrm{L}^{-1}$. Os fragmentos utilizados para a quantificação e identificação são apresentados na Tabela 2.

\section{RESULTADOS}

Apesar de ser uma técnica simples e versátil, a SPME necessita que haja a otimização dos parâmetros de extração para que seja obtido o melhor rendimento possível para cada tipo de matriz. Nesse sentido foram realizados estudos para se definir qual a melhor fibra, modo de extração, tempo de extração, velocidade de agitação, concentração iônica e $\mathrm{pH}$ da amostra para que fosse atingida a maior sensibilidade possível.

\section{Otimização dos parâmetros de extração}

\section{Escolha do tipo de fibra e modo de extração}

Para a escolha da fibra foram efetuados testes no modo "scan", onde as extrações foram realizadas nos modos direto e "headspace", utilizando as fibras PDMS, CAR/PDMS, CW/DVB e PA em soluções onde foram adicionadas concentrações conhecidas dos pesticidas em água Milli-Q, sob agitação constante (aproximadamente 900 rpm), temperatura ambiente e tempo de extração de $15 \mathrm{~min}$. No modo direto, a fibra foi inserida na fase líquida da amostra $(4 \mathrm{~mL})$ e no modo "headspace" o volume foi reduzido à metade e a fibra foi inserida na fase gasosa da amostra.

Na Figura 1 são apresentados os comportamentos dos diferentes tipos de fibra, em relação à área dos analitos estudados. Nenhuma das fibras avaliadas apresentou bons resultados para as extrações efetuadas no modo "headspace", no modo direto somente a fibra CAR/PDMS não apresentou bons resultados.

A fibra que apresentou o melhor desempenho foi a de PA, $85 \mu \mathrm{m}$, onde foram obtidas as melhores respostas para pentaclorofenol e atrazina, além de boas respostas para bifentrina e cis-permetrina.

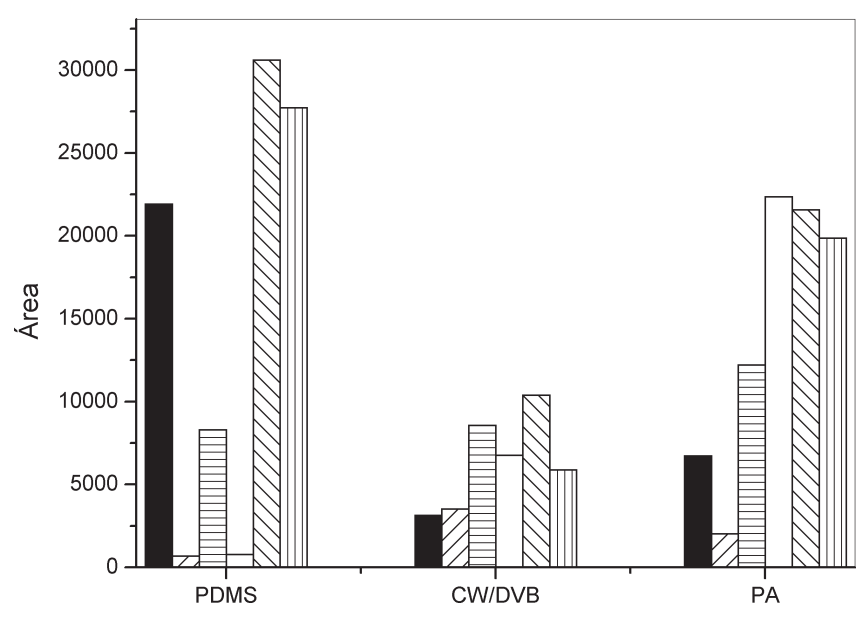

Figura 1. Perfil de extração no modo direto para algumas das fibras avaliadas. Resposta normalizada para $1 \mu \mathrm{g} L^{-1}$ para cada pesticida em água Milli- $Q$, extraídos sob agitação constante de 900 rpm e temperatura ambiente

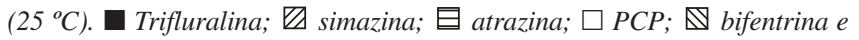
四 cis-permetrina 


\section{Tempos de extração}

Com a definição do tipo de fibra e do modo de extração foi avaliado o tempo de extração no intervalo de 5 a $90 \mathrm{~min}$, sob agitação constante. Os resultados obtidos são apresentados na Figura 2. Apesar dos bons resultados em relação às áreas obtidas dos analitos, mesmo após 90 min o equilíbrio não foi alcançado. Entretanto, para análise de rotina o equilíbrio não é necessário desde que as condições de extração sejam mantidas; portanto, para os estudos posteriores foi escolhido o tempo de $30 \mathrm{~min}$.

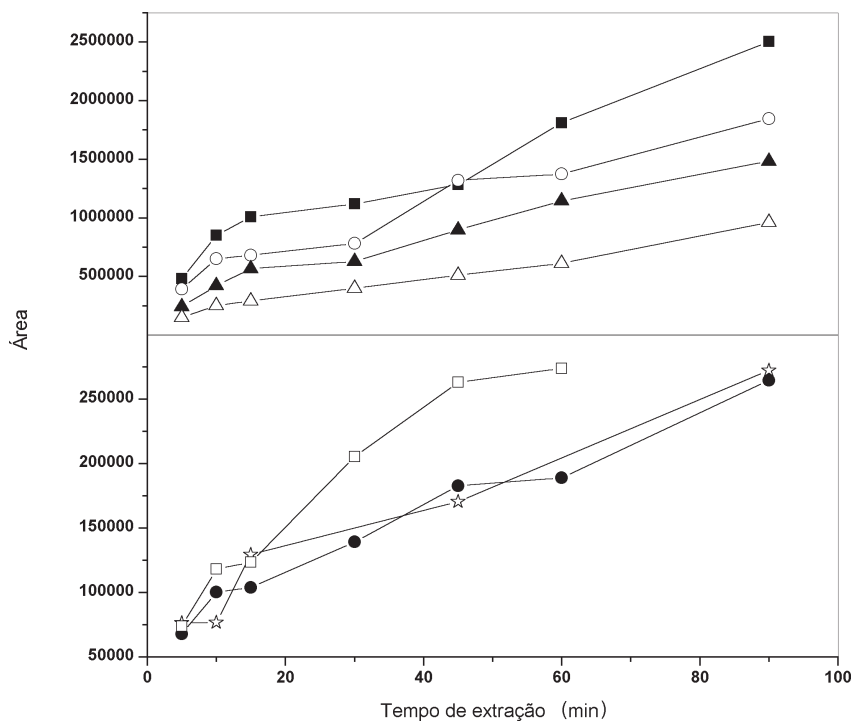

Figura 2. Estudo do tempo de extração dos pesticidas no modo SIM, com adição dos pesticidas em água Milli-Q, extraídos com imersão direta utilizando fibra de PA, sob agitação constante de 900 rpm e temperatura

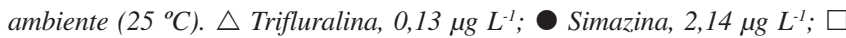
Atrazina, 1,03 $\mu \mathrm{g} \mathrm{L}^{-1}$; 蛇 Pentaclorofenol, 0,10 $\mu \mathrm{g} \mathrm{L}^{-1}$; $\mathbf{\square}$ Bifentrina, 0,09 $\mu \mathrm{g}$ $L^{-1}$; $\bigcirc$ cis-permetrina, $0,10 \mu g L^{-1}$ e $\boldsymbol{\Delta}$ trans-permetrina $0,10 \mu g L^{-1}$

\section{Efeito da velocidade de agitação}

A quantidade de analito extraído depende da velocidade na qual o processo de SPME alcança o equilíbrio através da razão de transferência de massa da fase aquosa para a fibra. Usualmente essa transferência de massa pode ser melhorada através da agitação ${ }^{21}$. A extração torna-se eficiente quando a agitação é utilizada para facilitar o transporte de massa entre a fase aquosa e a fibra ${ }^{11-13}$. A influência da velocidade de agitação foi determinada empregando-se a fibra de PA no modo direto com tempo de extração em 30 min. Para as faixas de velocidade de agitação estudadas (150 a 900 rpm) observou-se que a eficiência da extração foi melhor em velocidades de agitação de $900 \mathrm{rpm}$, exceto para atrazina e simazina onde não foi observada alteração da eficiência da extração em função da velocidade de agitação.

\section{Efeito da concentração salina}

A sensibilidade do método de extração freqüentemente aumenta na presença de sal na solução. Com o aumento da concentração iônica da fase aquosa, os analitos são conduzidos do seio da solução para a fibra, devido ao efeito "salting out"11-13. A influência da concentração iônica foi determinada empregando-se a fibra de PA no modo direto com tempo de extração em 30 min e velocidade de agitação de 900 rpm. Os resultados obtidos são apresentados na Figura 3. Dos compostos analisados, a trifluralina demonstrou um comportamento totalmente oposto aos demais, tendo-se obtido o maior valor de área sem a adição de sulfato de sódio. Já os piretróides apresentaram um comportamento peculiar para o perfil de extração com a presença de um mínimo ao redor de $5 \%$ de sal. Os perfis de extração para atrazina, simazina e pentaclorofenol aumentaram com o aumento da concentração salina, sendo que este efeito foi muito mais exacerbado para o pentaclorofenol que para os pesticidas triazínicos. Para os estudos seguintes foi adotada a concentração de $20 \% \mathrm{~m} / \mathrm{v}$ de $\mathrm{Na}_{2} \mathrm{SO}_{4}$.

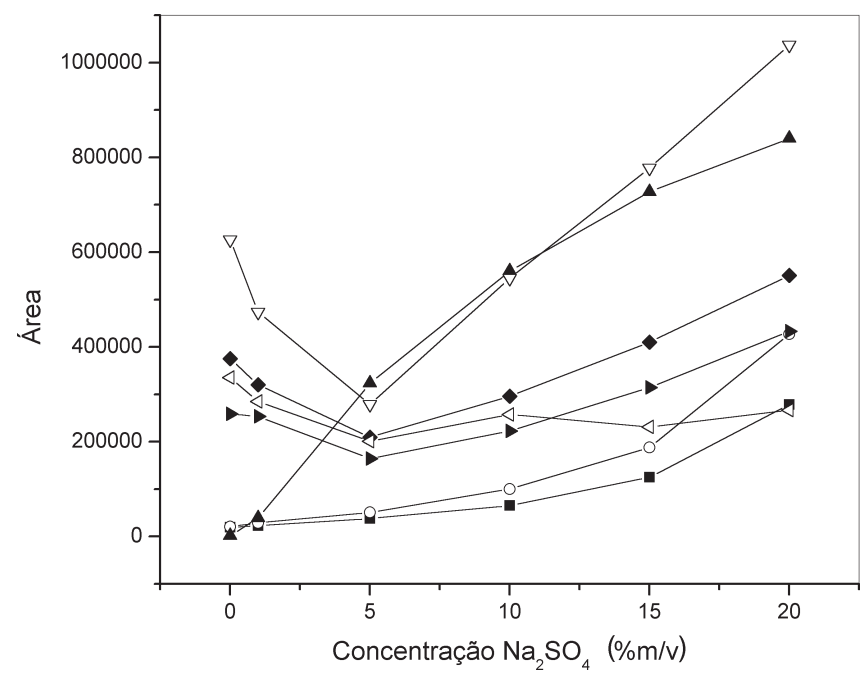

Figura 3. Efeito da concentração salina na extração no modo SIM, com adição dos pesticidas em água Milli-Q, extraídos durante 30 min com fibra de PA, sob agitação constante de 900 rpm e temperatura ambiente $\left(25^{\circ} \mathrm{C}\right)$. $\triangleleft$ Trifluralina, $0,13 \mu \mathrm{g} \mathrm{L}^{-1}$; $\mathbf{\square}$ Simazina, 2,18 $\mu \mathrm{g} \mathrm{L}^{-1}$; $\bigcirc$ Atrazina, 1,03 $\mu \mathrm{g} \mathrm{L}^{-1}$;

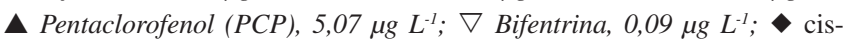
permetrina, $0,10 \mu \mathrm{g} L^{-1} e>$ trans-permetrina $0,10 \mu \mathrm{g} L^{-1}$

\section{Efeito do $\mathrm{pH}$}

Para compostos com grupamentos ácidos ou básicos, o método de extração para amostras aquosas no modo direto pode ser dificultado, devido à alta afinidade do analito com a matriz ${ }^{11-13}$.

A influência do $\mathrm{pH}$ foi determinada empregando-se a fibra de PA no modo direto com tempo de extração em $30 \mathrm{~min}$, velocidade de agitação de $900 \mathrm{rpm}$ e concentração salina de $20 \% \mathrm{~m} / \mathrm{v}$ de $\mathrm{Na}_{2} \mathrm{SO}_{4}$. Os resultados obtidos para o estudo com pentaclorofenol são apresentados na Figura 4. O pentaclorofenol, por possuir grupamentos protonáveis, foi o pesticida mais influenciado pela variação do $\mathrm{pH}$. Para os demais compostos estudados não houve variação representativa das áreas medidas nos diferentes valores de $\mathrm{pH}$ estudados. Embora o melhor valor de área tenha sido em $\mathrm{pH}=2$ para o pentaclorofenol, o $\mathrm{pH}=3$ foi escolhido para ser utilizado no método analítico por apresentar um valor de área melhor que em $\mathrm{pH}=4(20 \%$ menor que a área do $\mathrm{pH}=2$ ) para o pentaclorofenol, uma vez que em longo prazo $\mathrm{pH}=2$ pode ser prejudicial à fibra de $\mathrm{PA}$.

Portando o método analítico proposto foi definido com as seguintes condições: fibra de PA de $85 \mu \mathrm{m}$, volume de amostra $4 \mathrm{~mL}$; extração no modo direto, tempo de extração de $30 \mathrm{~min}$, velocidade de agitação $900 \mathrm{rpm}$, concentração iônica $20 \% \mathrm{~m} / \mathrm{v}$ de $\mathrm{Na}_{2} \mathrm{SO}_{4}$ e $\mathrm{pH} 3$.

\section{Efeito do "carryover"}

Por ser uma técnica de adsorção a SPME pode estar sujeita aos chamados "efeitos de memória"11 ou "carryover", que são caracterizados pela desorção incompleta dos analitos adsorvidos na fibra, que pode ser mais acentuada para compostos com alta afinidade ${ }^{12,13}$ pelo recobrimento da fibra. Os resultados obtidos para os efeitos de "carryover", em duas concentrações diferentes, para a metodologia 


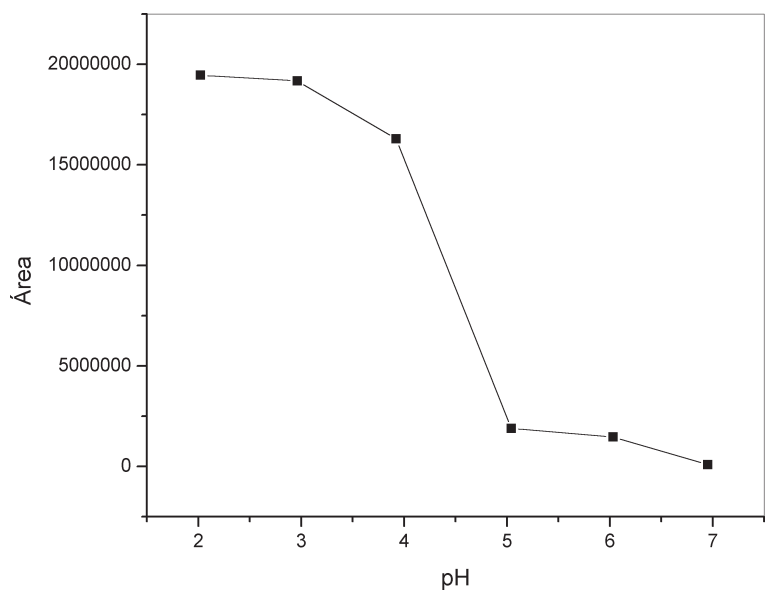

Figura 4. Efeito do pH na extração no modo SIM, com adição de Pentaclorofenol (PCP) 5,07 $\mu \mathrm{g} \mathrm{L}^{-1}$ em água Milli-Q, extraído durante 30 min com fibra de PA, sob agitação constante de 900 rpm, concentração salina de $20 \% \mathrm{~m} / \mathrm{v}$ de $\mathrm{Na}_{2} \mathrm{SO}_{4}$ e temperatura ambiente

proposta são apresentados na Figura 5. Como pode ser observado foram necessários cerca de nove ciclos de desorção para que os analitos fossem completamente extraídos da fibra. Dessa forma, para eliminar o efeito de "carryover" foi necessário deixar a fibra inserida no injetor durante toda a corrida cromatográfica para a remoção total dos analitos adsorvidos.

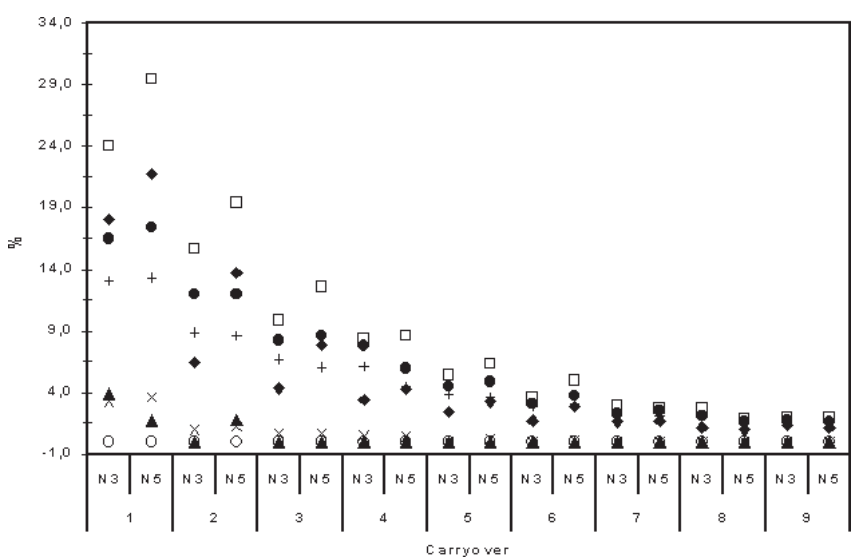

Figura 5. Efeito "carryover" na desorção para extração no modo SIM, com adição dos pesticidas em água Milli-Q, extraídos durante 30 min com fibra de PA, sob agitação constante de $900 \mathrm{rpm}$, concentração salina de $20 \% \mathrm{~m} / \mathrm{v}$ de $\mathrm{Na}_{2} \mathrm{SO}_{4}$ e temperatura ambiente $\left(25^{\circ} \mathrm{C}\right)$. A concentração para N3 foi 0,5 $\mu \mathrm{g} L^{-1}$ e para N5 foi $2 \mu \mathrm{g} L^{-1}$ para cada pesticida. Trifluralina; $\bigcirc$ Simazina; $\mathbf{\Delta}$ Atrazina; $\times$ Pentaclorofenol; $\square$ Bifentrina; - cis-Permetrina $e+$ trans-Permetrina

\section{Limite de detecção e recuperação}

Na Figura 6 apresentou-se o cromatograma reconstruído, no modo SIM, para os compostos estudados, obtido pela metodologia SPME proposta para uma concentração próxima ao limite de detecção (LD). Na Tabela 2 são apresentados os resultados obtidos para as curvas de referência (inclinação e intersecção), o coeficiente de correlação linear $\left(\mathrm{r}^{2}\right)$, o limite de detecção determinado para a relação S/ $\mathrm{N}=3$ e os fragmentos utilizados para quantificação dos pesticidas. O coeficiente de correlação linear, nas faixas de concentração avaliadas, foi maior que 0,99 e os limites de detecção obtidos apresentaram valores melhores que os recomendados por organismos mundiais $^{8}\left(0,1 \mu \mathrm{g} \mathrm{L}^{-1}\right)$.

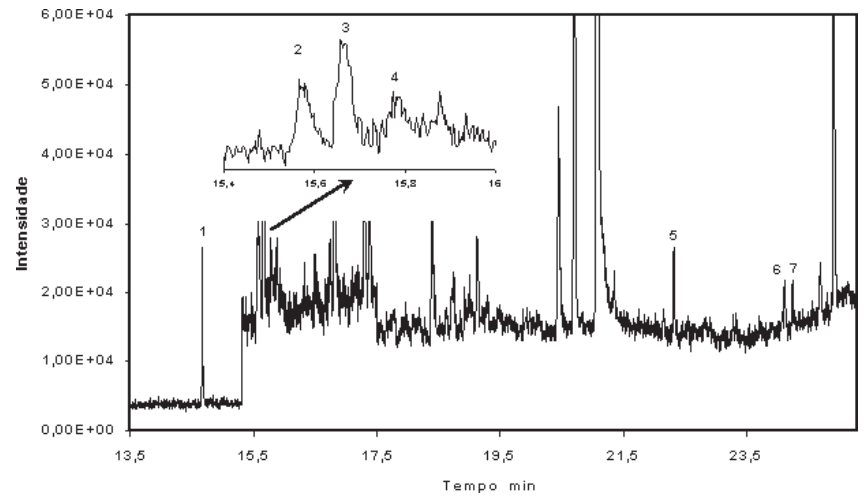

Figura 6. Cromatograma obtido no modo SIM com a metodologia proposta para o nível 1 de recuperação (Tabela 3). Identificação dos picos: 1 Trifluralina; 2 -Simazina; 3 -Atrazina; 4 - Pentaclorofenol; 5 -Bifentrina; 6 - cis-Permetrina e 7 - trans-Permetrina. Os demais picos não foram identificados

Os valores obtidos para o estudo de recuperação, em água Milli-Q, com o método proposto são apresentados na Tabela 3 e foram determinados três níveis de concentração diferentes. Como pode ser observado, os valores obtidos para as recuperações encontram-se na faixa de 90 a $110 \%$, atestando o bom desempenho da metodologia proposta, mesmo em concentrações muito próximas ao limite de detecção.

\section{Determinação em amostras de água}

A metodologia proposta neste trabalho foi aplicada em uma amostra de água bruta coletada em Cananéia. Na Figura 7a é apresentado o cromatograma reconstruído no modo SIM obtido para esta amostra. Apesar de ser uma região com intensa atividade agrícola, não foram detectadas presenças dos pesticidas estudados.

Para verificar a eficácia do método proposto foi realizado um estudo onde foi adicionada a concentração de $0,1 \mu \mathrm{g} \mathrm{\textrm {L } ^ { - 1 }}$ para cada

Tabela 2. Limite de detecção obtido para a metodologia proposta

\begin{tabular}{lllccc}
\hline Pesticida & Fragmento $(\mathrm{m} / \mathrm{z})$ & Inclinação & Intersecção & Correlação & LD $\left(\mu \mathrm{g} \mathrm{L}^{-1}\right)$ \\
\hline Trifluralina & $306^{*} ; 307 ; 264$ & $4,68 \times 10^{5}$ & $3,59 \times 10^{4}$ & 0,9979 & 0,006 \\
Simazina & $201^{*} ; 203 ; 186$ & $1,95 \times 10^{4}$ & $1,23 \times 10^{4}$ & 0,9932 & 0,10 \\
Atrazina & $200^{*} ; 215 ; 217$ & $7,12 \times 10^{4}$ & $2,59 \times 10^{4}$ & 0,9977 & 0,04 \\
Pentaclorofenol & $266^{*} ; 264 ; 268$ & $1,12 \times 10^{6}$ & $1,37 \times 10^{5}$ & 0,9929 & 0,004 \\
Bifentrina & $181^{*} ; 182 ; 165$ & $4,93 \times 10^{5}$ & $4,49 \times 10^{4}$ & 0,9914 & 0,005 \\
cis-Permetrina & $183^{*} ; 184 ; 163$ & $4,05 \times 10^{5}$ & $3,28 \times 10^{4}$ & 0,9959 & 0,010 \\
trans-Permetrina & $183^{*} ; 184 ; 163$ & $3,55 \times 10^{5}$ & $3,75 \times 10^{4}$ & 0,9964 & 0,010 \\
\hline
\end{tabular}

* - fragmentos utilizados para quantificação; os demais foram utilizados para confirmação 
Tabela 3. Recuperação pela metodologia proposta

\begin{tabular}{|c|c|c|c|c|c|c|}
\hline \multirow[b]{2}{*}{ Pesticida } & \multicolumn{2}{|c|}{ Nível 1} & \multicolumn{2}{|c|}{ Nível 2} & \multicolumn{2}{|c|}{ Nível 3} \\
\hline & Conc. $\left(\mu \mathrm{g} \mathrm{L}^{-1}\right)$ & $\% \operatorname{Rec}^{\mathrm{a})}$ & Conc. $\left(\mu \mathrm{g} \mathrm{L}^{-1}\right)$ & $\% \operatorname{Rec}^{\mathrm{a})}$ & Conc. $\left(\mu \mathrm{g} \mathrm{L}^{-1}\right)$ & $\% \operatorname{Rec}^{\mathrm{a})}$ \\
\hline Trifluralina & 0,020 & $98 \pm 13$ & 0,060 & $97 \pm 8$ & 0,180 & $96 \pm 9$ \\
\hline Simazina & 0,400 & $101 \pm 12$ & 1,200 & $100 \pm 9$ & 3,600 & $98 \pm 8$ \\
\hline Atrazina & 0,080 & $107 \pm 17$ & 0,240 & $104 \pm 13$ & 0,720 & $97 \pm 7$ \\
\hline Pentaclorofenol & 0,004 & $103 \pm 10$ & 0,012 & $97 \pm 9$ & 0,036 & $95 \pm 15$ \\
\hline Bifentrina & 0,010 & $100 \pm 24$ & 0,030 & $93 \pm 16$ & 0,090 & $106 \pm 22$ \\
\hline Cis-Permetrina & 0,010 & $94 \pm 16$ & 0,030 & $98 \pm 6$ & 0,090 & $95 \pm 17$ \\
\hline Trans-Permetrina & 0,010 & $100 \pm 11$ & 0,030 & $100 \pm 9$ & 0,090 & $108 \pm 22$ \\
\hline
\end{tabular}

a) Resultados para 3 determinações, (média \pm desvio padrão)

pesticida, recomendado pela Comunidade Européia como o máximo permitido para água potável ${ }^{14}$, na mesma amostra de água bruta coletada em Cananéia. Na Figura $7 b$ é apresentado o cromatograma reconstruído no modo SIM obtido para o estudo. Os resultados para recuperação obtidos para a média de três determinações efetuadas em dias diferentes foram trifluralina $(106 \pm 13) \%$, simazina $(110 \pm 10) \%$, atrazina $(103 \pm 20) \%$, pentaclorofenol $(132 \pm 18) \%$, bifentrina $(98 \pm 8) \%$ e cis-permetrina $(93 \pm 15) \%$.
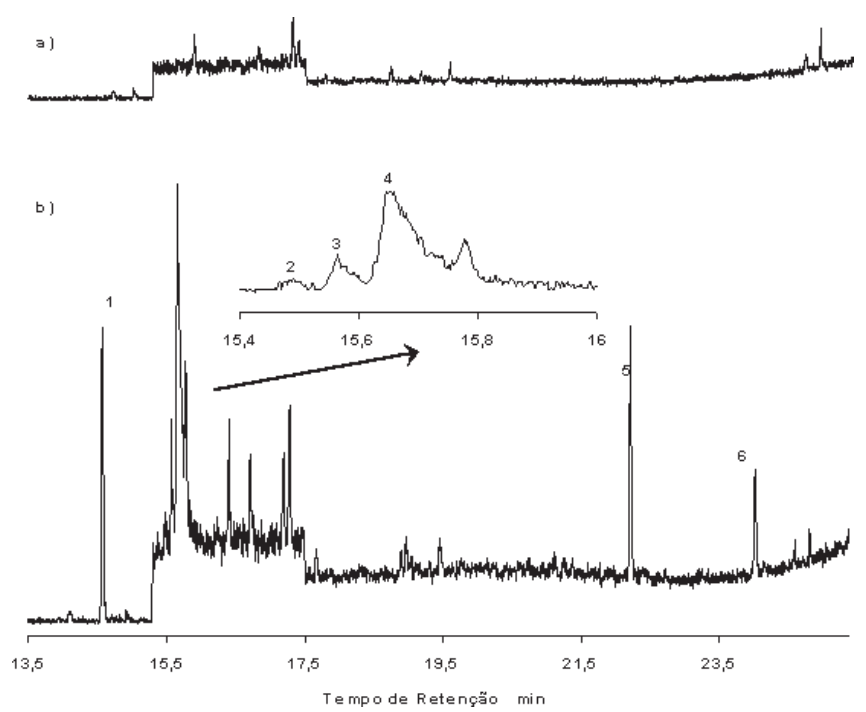

Figura 7. a) Cromatograma obtido no modo SIM para amostra coletada em Cananéia; b) cromatograma obtido no modo SIM para amostra coletada em Cananéia com adição de 0,1 $\mu \mathrm{g} \mathrm{L}^{-1}$ de cada pesticida. Identificação dos picos: 1 -Trifluralina; 2 -Simazina; 3 -Atrazina; 4 -Pentaclorofenol; 5 - Bifentrina e 6 - cis-Permetrina. Os demais picos não foram identificados

\section{CONCLUSÕES}

O método analítico desenvolvido nesse estudo, utilizando SPMEGC/MS para a determinação dos pesticidas trifluralina, simazina, atrazina, pentaclorofenol, bifentrina, cis-permetrina e transpermetrina mostrou ser simples, rápido e sensível. Dentre suas principais vantagens pode ser destacada a pequena quantidade de amostra necessária para as determinações $(4 \mathrm{~mL})$, o que pode ser útil para amostras onde o volume é limitado.
O método proposto apresentou faixas para recuperação, com boa reprodutibilidade, dentro dos intervalos recomendados por diferentes critérios de validação, mesmo em concentrações muito próximas ao limite de detecção. Por outro lado, os valores encontrados para os limites de detecção foram, no geral, cerca de uma ordem de grandeza inferior aos sugeridos $\left(0,1 \mu \mathrm{g} \mathrm{L}^{-1}\right)$ por organismos de regulação mundial para determinação de pesticidas em água potável.

\section{AGRADECIMENTOS}

Os autores agradecem ao CNPq pelo auxilio concedido (processo $n^{\circ}$ 470150/01-7) e à Dra. M. A. F. Pires pelo uso do CG/MS. E. Komatsu agrade ao $\mathrm{CNPq}$ pela bolsa de mestrado concedida.

\section{REFERÊNCIAS}

1. Larini, L.; Toxicologia dos Praguicidas, Manole: São Paulo, 1999.

2. Biziuk, M.; Przyjazny, A.; Czerwinski, J.; Wiergowski, M.; J. Chromatogr. 1996, 754, 103.

3. Younes, M.; Galal-Gorchev, H.; Food Chem. Toxicol. 2000, 38, S87.

4. http://www.epa.gov/pesticides/about/, acessada em Abril 2003.

5. Holf, R.; Zoonen, P.; J. Chromatogr. 1999, 843, 301.

6. Clive, T.; The Pesticide Manual - Incorporating The Agrochemicals Handbooks, 10 ${ }^{\text {th }}$ ed., Crop Protection Publications: United Kingdom, 1994.

7. Bruzzoniti, M. C.; Sarzanini, C.; Mentasti, E.; J. Chromatogr. 2000, 902, 289.

8. Balinova, A.; J. Chromatogr. 1996, 754, 125.

9. Tekel, J.; Hatrík, S.; J. Chromatogr. 1996, 754, 397.

10. Hoff, G. R.; Zoonen, P.; J. Chromatogr. 1999, 843, 301.

11. Wercinski, S. A. S.; Solid Phase Microextraction - A Practical Guide, Marcel Dekker: USA, 1999.

12. Pawliszyn, J.; Solid Phase Microextraction - Theory and Practice, WileyVCH: USA, 1997

13. Dugay, J.; Miège, C.; Hennion, M. C.; J. Chromatogr. 1998, 795, 27.

14. Krutz, L. J.; Senseman, S. A.; Sciumbato, A. S.; J. Chromatogr. 2003, 999, 103.

15. Goncalves, C.; Alpendurada, M. F.; J. Chromatogr. 2002, 963, 19.

16. Eisert, R.; Levsen, K.; J. Chromatogr. 1996, 733, 143.

17. Kataoka, H.; Lord, H. L.; Pawliszyn, J.; J. Chromatogr. 2000, 880, 35.

18. Beltran, J.; López, F. J.; Hernández, F.; J. Chromatogr. 2000, 885, 389.

19. Natangelo, M.; Tavazzi, S.; Fanelli, R.; Benfenati, E.; J. Chromatogr. 1999, $859,193$.

20. Aguilar, C.; Peñalver, S.; Pocorull, E.; Marcé, R. M.; J. Chromatogr. 1998, $795,105$.

21. Lambropoulou, D. A.; Konstantinou, I. K.; Albanis, T. A.; J. Chromatogr. 2000, 893, 143.

22. Lambropoulou, D. A.; Konstantinou, I. K.; Albanis, T. A.; J. AOAC Int. 2002, 85, 486

23. Resolução CONAMA nº 20 de 18/06/1986. 